\title{
Rechtliche Anforderungen an ein System zur Erreichbarkeitssteuerung
}

\author{
Technische Umsetzung abstrakter rechtlicher Vorgaben an \\ ein Erreichbarkeitsmanagement-System im Arbeitskontext
}

Nadine Miedzianowski

\section{Zusammenfassung}

Die zunehmende Verbreitung moderner Informations- und Kommunikationstechnologien prägt und verändert die Arbeitswelt. Vermehrt sehen sich Unternehmen und Beschäftigte daher mit dem Problem der ständigen technikbedingten Erreichbarkeit konfrontiert. Solche Technologien bieten neben Risiken, aber auch die Chance zur effektiven Gestaltung eines Erreichbarkeitsmanagements. Als technische Lösung wurde im Projekt „SANDRA“ ein System zur Erreichbarkeitssteuerung entwickelt. Der Beitrag geht der Frage nach, welche rechtlichen Anforderungen an ein solches System zu stellen sind, das im Arbeitskontext zur Anwendung kommt, und welche technischen Anforderungen aus den teils abstrakten und unbestimmten rechtlichen Vorgaben abgeleitet werden können. Neben Bestimmungen aus dem Arbeits- und Betriebsverfassungsrecht, sind aufgrund der Daten, die bei der Nutzung eines technischen Systems verarbeitet werden, vor allem Vorgaben des Datenschutzrechts und der Datensicherheit ausschlaggebend.

\subsection{Einleitung}

Die Gestaltung und Organisation des Arbeitslebens und die Aufgabenfelder vieler Berufe sind stark durch Informations- und Kommunikationstechnologien, das Internet und verschiedene Kommunikationssysteme gekennzeichnet. Charakteristisch für die Digitalisierungsprozesse

\footnotetext{
N. Miedzianowski $(\bowtie)$

Projektgruppe verfassungsverträgliche Technikgestaltung (provet), Universität Kassel, Kassel, Deutschland

E-Mail: n.miedzianowski@uni-kassel.de
} 
sind sog. Mobile-Devices - mobile Endgeräte wie Smartphones, Wearables oder Tablet PCs -, die durch ihre steigende Leistungsfähigkeit und Ortsunabhängigkeit neue Möglichkeiten für die Gestaltung des beruflichen Alltags eröffnen. Sie gehen mit neuen Kommunikationsformen einher und ermöglichen mittels E-Mail, SMS und Messenger-Nachricht eine einfache und schnelle Kontakt- und Kommunikationsanfrage. Solche technischen Errungenschaften können den Arbeitsalltag erleichtern, indem sie zu mehr Flexibilität und Autonomie bei der individuellen Arbeitszeitgestaltung führen und einen ortsunabhängigen Zugriff auf Informationen ermöglichen. Sie bergen jedoch auch Risiken wie potenzielle Überlastung aufgrund einer dauerhaften Kommunikationsbereitschaft oder das Vermischen von Arbeits- und Freizeit (s. zu den Chancen und Risiken auch Kap. 3). Ein zentrales Problem ist dabei die daraus resultierende Gefahr der ständigen technikbedingten Erreichbarkeit. Aus beruflichen Kontaktanfragen ergeben sich regelmäßig berufliche Tätigkeiten, die nicht nur darin bestehen, die Kommunikationsanfragen zu beantworten. Die Problematik und die daraus entstehenden Risiken für Beschäftigte haben bereits einige Unternehmen erkannt. Die Lösungen sind meist jedoch nicht für alle Beschäftigte praktikabel und können dem Problem ständiger Erreichbarkeit nur bedingt durch eine zeitweilige Unterbrechung der Kommunikationsverbindungen entgegenwirken (Daimler 2014; Spiegel Online 2011). Neben organisatorischen Maßnahmen zur Bewältigung technikbedingter Erreichbarkeit kann die Entwicklung einer technischen Lösung ein erfolgreiches Erreichbarkeitsmanagement ermöglichen. Welche Chancen und Risiken aus rechtlicher Sicht aus der Verwendung einer solchen Technologie im Arbeitskontext prognostiziert werden können und welche rechtlichen Anforderungen daraus für die Technikgestaltung resultieren, wird im nachfolgenden Beitrag beleuchtet.

\subsection{Chancen und Risiken eines Erreichbarkeitsmanagement-Systems}

Im Rahmen des vom Bundesministerium für Bildung und Forschung (BMBF) und dem Europäischen Sozialfonds (ESF) geförderten Projekts „Gestaltung der Arbeitswelt der Zukunft durch Erreichbarkeitsmanagement“ (SANDRA) wurde ein System zur Erreichbarkeitssteuerung entwickelt (s. zum Projekt Miedzianowski 2017; Laufs et al. 2018; Miedzianowski et al. 2019). Das Erreichbarkeitsmanagement-System (EMS) wird in eine App für das Smartphone sowie eine Serverkomponente unterteilt (s. zum System ausführlich Kap. 5). Anhand der App können Beschäftigte unterschiedliche Erreichbarkeitseinstellungen vornehmen (z. B. individuelle Erreichbarkeitszeiträume, Erreichbarkeit abhängig von Ereignissen wie einem Meeting oder Erreichbarkeit für Personen und Personengruppen) und dadurch eingehende Kommunikationsversuche entsprechend ihrer persönlichen Erreichbarkeitspräferenzen steuern. Zudem kann die Funktion einer selbstlernenden Komponente zugeschaltet werden, die eigenständig mittels E-Mail-Informationen über die Zustellung einer E-Mail entscheidet. Bestehende Unternehmensregelungen zur beruflichen Erreichbarkeit, spezifische Arbeitszeitregelungen oder Betriebsvereinbarungen des Ein- 
satzunternehmens werden im Basisregelwerk des Systems abgebildet, sodass es den unterschiedlichen Anforderungen des Einsatzunternehmens gerecht werden kann.

\subsubsection{Chancen eines Erreichbarkeitsmanagement-Systems}

Mit dem Einsatz eines EMS sind viele Erwartungen verbunden, den Arbeitsalltag für Beschäftigte angenehmer zu gestalten sowie das Zusammenspiel zwischen Arbeits- und Freizeit deutlich zu verbessern. Das Ziel ist es, die Lebensqualität und Zufriedenheit der Beschäftigten zu erhöhen und dadurch aus Unternehmenssicht schließlich auch die Produktivität.

Mit Hilfe des Systems wird den Beschäftigten eine eigene Zeitgestaltung ermöglicht, indem sie eigenständig und flexibel ihre Ruhephasen, Zeiten des unterbrechungsfreien Arbeitens sowie Erreichbarkeitseinstellungen außerhalb ihrer regulären Arbeitszeit einstellen können. Da sie nicht durch eingehende Telefonanrufe oder E-Mails abgelenkt werden, können sie sich konzentriert ihrer Arbeit widmen. Auf diese Weise ist ein ruhigeres und damit produktiveres Arbeiten möglich. Diese Optionen vereinfachen zudem die Grenzziehung zwischen dem beruflichen und privaten Lebensbereich der Mitarbeiter, die diese Trennung wünschen (s. hierzu auch Kap. 3). Eine selbstbestimmte Arbeitsgestaltung führt schließlich zu einer verbesserten Vereinbarkeit von Beruf und Privatleben (Huber 2016, S. 88; Bundesanstalt für Arbeitsschutz und Arbeitsmedizin 2017, S. 57), sodass dahingehend mit positiven Auswirkungen für die Work-Life-Balance der Systemnutzer gerechnet werden kann. Denn eine bessere Vereinbarkeit beider Bereiche wird ermöglicht, wenn Beschäftigte selbstbestimmte Arbeitszeiten sowie die Möglichkeit zur Flexibilisierung von Arbeitszeit und -ort auch tatsächlich durchsetzen können (vgl. hierzu Hassler et al. 2016, S. 13; IG Metall 2017, S. 32). Dies ist insbesondere vor dem Hintergrund der veränderten Arbeitsbedingungen und -verhältnisse von Bedeutung und begünstigt die Planbarkeit der Arbeitszeit. Vor allem in projektbezogenen Arbeitsverhältnissen ist Flexibilität von hoher Bedeutung. Das System ermöglicht somit eine bessere autonome Gestaltung der eigenen Arbeitszeit bezogen auf die Dauer und die Verteilung der Arbeit und fördert eine selbstbestimmte zeitliche Flexibilität von Beschäftigten in unterschiedlichen Beschäftigungsverhältnissen. Dadurch wird schließlich die berufliche Beanspruchung und der Stress der Beschäftigten nicht nur während ihres Arbeitsalltags, sondern auch in ihrer Freizeit gemindert. Bereits kurzzeitige Unterbrechungen durch Telefonanrufe oder Nachrichten führen nämlich zu einer Unterbrechung der Erholungszeit, wobei gerade eine durchgängige Ruhezeit für die Erholung der Beschäftigten notwendig ist (Strobel 2013, S. 16). Dabei ist der positive Zusammenhang zwischen der guten Gestaltung der Arbeitszeit und einer besseren psychischen Gesundheit der Beschäftigten zu berücksichtigen (Bundesanstalt für Arbeitsschutz und Arbeitsmedizin 2017, S. 57). Zudem unterstützt ein System zur Erreichbarkeitssteuerung seine Nutzer auch bei der Gestaltung der eigenen Freizeit, was insbesondere für Arbeitsverhältnisse mit unregelmäßigen Arbeitszeiten als 
Chance anzusehen ist. Durch eine gezielte Abgrenzung der Arbeitszeit von der Freizeit ist die Zeit für die Familie, die Pflege von Angehörigen oder private Aktivitäten planbarer.

Auf der Arbeitgeberseite soll ein solches System zu einer Verbesserung des Arbeitsumfelds und der Unternehmensproduktivität führen, indem die positiven Effekte der einzelnen Beschäftigten (z. B. erhöhtes Wohlbefinden, Stressreduktion, Produktivitätssteigerungen durch unterbrechungsfreie Arbeitsphasen) auf das Einsatzunternehmen ausstrahlen und sich nutzbringend auf dieses auswirken. So können viele der individuellen Chancen des Systems zu einer höheren Beschäftigtenzufriedenheit beitragen sowie negative gesundheitliche Folgen der dauerhaften Erreichbarkeit senken, was sich positiv auf die Anzahl krankheitsbedingter Fehltage auswirken kann (vgl. Arlinghaus und Nachreiner 2013, S. 1200; Lindecke 2015, S. 31). Bestehen im Unternehmen keine Regelungen dazu, wann und für wen die Mitarbeiter außerhalb ihrer Arbeitszeit für berufliche Belange erreichbar sein sollen, kann es schnell zu Missverständnissen sowie falschen Erwartungshaltungen an die Erreichbarkeit der Beschäftigten kommen. Durch den Einsatz eines EMS können bezüglich der Erreichbarkeit klare Regeln abgebildet werden. Dies erleichtert das Durchsetzen betrieblicher Regelungen und verbessert das Arbeitsklima, da der Arbeitgeber die Problematik ständiger Erreichbarkeit ernst nimmt und Zeiträume der Unerreichbarkeit Akzeptanz finden.

\subsubsection{Risiken eines Erreichbarkeitsmanagement-Systems}

Neben möglichen Chancen birgt der Einsatz eines EMS auch potenzielle Risiken. Vorrangig werden sich diese für die Beschäftigten als Systemverwender ergeben und sich auf diese auswirken. Für die Beurteilung sind die Zugriffsmöglichkeiten auf das System, die bei der Verwendung anfallenden Daten und die technische Ausgestaltung des Systems ausschlaggebend.

Damit das System die Kommunikationsversuche der Beschäftigten regulieren kann, benötigt es eine große Menge an Daten und Informationen über seine Nutzer. Zum einen fallen bereits durch den Systemgebrauch verschiedene Daten, wie Verbindungsdaten und Inhaltsdaten aus beispielsweise eingehenden E-Mails oder Kalendereinträgen, an. Zum anderen erstellen die Beschäftigten im System selbstständig eine Art Profil über ihre Erreichbarkeitswünsche und ihre Erreichbarkeitssituation (z. B. Beginn und Ende der Arbeitszeit). Auf diese und weitere Informationen über die Beschäftigten greift das System zur Entscheidungsfindung und Steuerung eingehender Kommunikationsversuche zu. Zudem eröffnet das Zuschalten der selbstlernenden Systemkomponente zur intelligenten und bedarfsgerechten Zustellung von Kommunikationsversuchen die Möglichkeit der Informationsgewinnung über die Kommunikationsinhalte (s. hierzu Kap. 5).

Mit Hilfe des technischen Systems und den bei der Systemnutzung anfallenden Daten wäre es dem Arbeitgeber oder Dritten somit möglich, dieses zur Überwachung der Beschäftigten zu missbrauchen und die Daten zweckentfremdet zu nutzen. Mit Hilfe der 
Daten können ein Bewegungs-, Kommunikations- und Persönlichkeitsprofil über den Systemnutzer erstellt werden. Solche Profile lassen umfangreiche Rückschlüsse über diesen zu und sind nicht nur auf seine berufliche Situation beschränkt, da der Beschäftigte das System auch für seine private Kommunikation nutzen kann und sein Smartphone in der Regel nicht nur zu beruflichen Zwecken verwendet. Aus den spezifischen Systemeinstellungen können Informationen über die Persönlichkeit des Nutzers gewonnen oder es kann auf seine persönlichen Merkmale und Präferenzen geschlossen werden. Vor dem Hintergrund der privaten Nutzung besteht daneben die Gefahr, dass es zu einer allgegenwärtigen und dauerhaften Beobachtung des Beschäftigten durch den Arbeitgeber kommen könnte. Die dabei gewonnenen Informationen stehen gerade nicht im Zusammenhang mit seiner Arbeitsleistung. Daraus resultiert für den Systemnutzer ein Kontrollverlust über die eigenen Daten, da er nicht eigenständig festlegen und kontrollieren kann, inwiefern das System seine persönlichen Daten verwendet, an welchem Ort es die Informationen verarbeitet und analysiert und wer auf die Daten zugreifen kann.

Aus der möglichen Überwachung könnten ferner Informationen zur Anwesenheits-, Leistungs- und Verhaltenskontrolle herangezogen werden. Mit solchen Informationen kann einfach überprüft werden, ob die Beschäftigten ihren Arbeitspflichten nachkommen und entsprechend ihre Arbeitsleistung erbringen. Dadurch könnten sie das Gefühl bekommen, dauerhaft unter Beobachtung des Arbeitgebers zu stehen. Das Gefühl des Überwachtwerdens führt schließlich zu einer Beeinflussung des Verhaltens der Beschäftigten, sodass sie z. B. die Dauer eines Termins so anpassen, dass ihre Anwesenheit mit den Vorstellungen des Arbeitgebers konform ist. Dadurch kann bei ihnen ein psychischer Anpassungsdruck erzeugt werden, durch den sie nicht mehr ihr Handeln selbstbestimmt gestalten, sondern dabei gehemmt werden (vgl. BAG 2008, S. 1189). Dieses Gefühl führt schließlich zu Verunsicherungen bei den Beschäftigten und kann sich negativ auf das Betriebsklima auswirken. Letzteres wird zudem durch die Erreichbarkeitseinstellungen der Beschäftigten beeinflusst, indem sie wahrnehmen, dass einige Mitarbeiter von ihren Kollegen nur während ihrer Arbeitszeit erreichbar sind, bestimmte Personen diese aber jederzeit kontaktieren können. Dies lässt Rückschlüsse darauf zu, in welcher Beziehung der Systemnutzer zu solchen Kontakten steht und welche er eventuell gegenüber anderen Personen bevorzugt.

Durch die Nutzung und den Einsatz eines EMS im Arbeitskontext können sich auch Risiken für den Arbeitgeber ergeben. Diese beschränken sich jedoch größtenteils auf die Technik selbst. Im Zusammenhang mit der Hauptfunktion des Systems, Kommunikationsversuche verzögert oder nicht zuzustellen, können durch einen unvorhersehbaren technischen Fehler, eine falsche Nutzung des Systems oder eine fehlerhafte Kategorisierung der Nachrichten und Anrufe durch den selbstlernenden Teil des Systems wichtige Kommunikationsversuche den Beschäftigten nicht oder zu einem zu späten Zeitpunkt erreichen. Eine fehlerhafte Zustellung wichtiger Kommunikationsinhalte führt nicht nur seitens des Arbeitgebers zu negativen Folgen wie verzögerter Leistungserbringung durch seine Mitarbeiter oder nicht fristgerechten Arbeitsergebnissen, sondern kann darüber hinaus bei den 
Nutzern zu einem Vertrauensverlust gegenüber der Technik führen und dessen Einsatz gefährden. Zudem besteht bei jedem technischen System das Risiko einer mangelnden Datensicherheit. Auch bei einem EMS besteht somit das Risiko, dass die im System hinterlegten Daten durch Dritte missbraucht und ausspioniert werden könnten. Auf diese Weise könnten auch Unternehmensgeheimnisse abgegriffen werden. So kaufen etwa unbefugte Personen Daten ein, die Unternehmensgeheimnisse enthalten.

Schließlich ist jedoch anzumerken, dass ein System zur Verbesserung der beruflichen Erreichbarkeitssituation vor allem durch solche Arbeitgeber eingesetzt wird, die gerade kein Kontrollinteresse gegenüber ihren Mitarbeitern haben. Es kann davon ausgegangen werden, dass insbesondere moderne Arbeitgeber, deren Unternehmen durch dynamische und vertrauensvolle Arbeitsstrukturen gekennzeichnet sind, solche Systeme in ihren Unternehmen einsetzen möchten. Trotzdem ist ein EMS grundsätzlich geeignet zur Kontrolle und Überwachung der Systemnutzer verwendet zu werden. So kann nicht ausgeschlossen werden, dass dieses durch den Arbeitgeber oder Dritte zur Überwachung der Beschäftigten genutzt wird und folglich nicht nur zum Managen eingehender Kommunikationsversuche. Auch wenn das System eine Kontrolle der Beschäftigten potenziell ermöglicht, ist dies jedoch stets von den Zugriffsmöglichkeiten des Arbeitgebers und Dritter auf das System und dessen Ausgestaltung abhängig.

\subsection{Rechtliche Vorgaben und Anforderungen}

Um eine rechtskonforme Anwendung eines EMS zu gewährleisten, müssen bei der Gestaltung des Systems rechtliche Vorgaben eingehalten werden. Durch den Einsatz des Systems im Arbeitskontext, sind neben arbeitsrechtlichen Bestimmungen aufgrund der Verarbeitung von Daten auch das Datenschutzrecht zu berücksichtigen. Diesen Rechtsnormen können jedoch keine konkreten technikbezogenen Vorgaben an ein solches spezifisches System entnommen werden. Problematisch ist zudem, dass sie im Laufe der Zeit Überarbeitungsprozesse durchlaufen und daher nur für ihre Gültigkeitsdauer angewandt werden können. Anders verhält es sich bei Rechtsnormen der obersten Ebene der Rechtshierarchie. Diese sind durch ihre dauerhafte Gültigkeit gekennzeichnet, sind allgemeingültig und regeln das Zusammenleben der Menschen. Jedoch sind verfassungsrechtliche Vorgaben sehr allgemein und generalklauselartig formuliert. Trotzdem wird immer wieder aus ihnen in Gerichtsentscheidungen ein technischer Bezug hergestellt wie beispielsweise durch das Bundesverfassungsgericht, das im sog. Volkszählungsurteil das Recht auf informationelle Selbstbestimmung aus dem allgemeinen Persönlichkeitsrecht des Grundgesetztes ableitete, um der Gefahr moderner Techniken der Datenspeicherung, -verarbeitung und -vernetzung entgegenzuwirken (BVerfG 1983).

Die mit den Rechtsnormen der obersten Ebene der Rechtshierarchie verbundenen sozialen Regelungsziele werden durch den Einsatz eines Techniksystems gefährdet oder gefördert (Hammer et al. 1993, S. 45 f.). Daraus können Chancen und Risiken für die von 
dem technischen System Betroffenen abgeleitet werden (Abschn. 4.2), sodass ermittelt werden kann, welche verfassungsrechtlichen Vorgaben dabei betroffen und für die Systemgestaltung von Relevanz sind. Auf diese Weise ist es möglich, rechtliche Anforderungen an technische Systeme aus allgemeinen Normen des Verfassungsrechts abzuleiten. Das Ziel muss es dabei sein, mittels der Anforderungen die aus dem Gebrauch eines EMS ermittelten Chancen zu fördern und die Risiken zu minimieren.

\subsubsection{Verhältnis zwischen nationalem und europäischem Recht}

Bei der Gestaltung eines EMS sind sowohl Vorgaben des nationalen Rechts als auch des Unionsrechts zu berücksichtigen. Das Unionsrecht zeichnet sich dadurch aus, dass Verordnungen unmittelbar gelten und sich nicht nur an die Organe und Mitgliedstaaten der Europäischen Union richten, sondern auch für Bürger der Union und Unternehmen Rechte und Pflichten begründen (vgl. Biervert, in: Schwarze et al. 2012: Art. 288 AEUV, Rn. 5). Als autonome Rechtsordnungen bestehen das Recht der Mitgliedstaaten und das der Union nebeneinander, sodass sich Konflikte zwischen diesen ergeben können. Im Verhältnis zum nationalen Recht genießt das Unionsrecht daher immer dann Anwendungsvorrang, wenn sich nationale und europäische Regelungen widersprechen (Roßnagel, in: ders. 2018, S. 42 ff.).

Für das deutsche Rechtssystem ist das Grundgesetz für die Bundesrepublik Deutschland (GG) mit seinen Grundrechten kennzeichnend. Sie prägen aufgrund ihres Verfassungsrangs die gesamte Rechtsordnung und entfalten dadurch umfassend ihre Rechtswirkung (Herdegen, in: Maunz und Dürig 2019: Art. 1 GG, Rn. 4). Sie regeln das menschliche Zusammenleben und beinhalten Wahrnehmungsvoraussetzungen, damit sie ihre Freiheitsgarantien gewährleisten und von den Grundrechtsträgern praktisch wahrgenommen werden können (Kirchhoff, in: Merten und Papier 2004: $§ 21$ Grundrechtsinhalte und Grundrechtsvoraussetzungen, Rn. 7 f.). Als Mitgliedstaat der Europäischen Union ist Deutschland aber auch an die Grundrechte der Europäischen Union gebunden, die durch die Charta der Grundrechte der Europäischen Union (GRCh) vorgegeben werden. Gemäß Art. 51 Abs. 1 Satz 1 GRCh gilt die Charta für die Organe, Einrichtungen und sonstige Stellen der Union sowie für die Mitgliedstaaten ausschließlich bei der Durchführung von Unionsrecht. Somit ist sie bei der Durchführung des Unionsrechts, wie z. B. bei der Vollziehung von Verordnungen oder bei der Umsetzung von Richtlinien, unmittelbar anzuwenden (Starke 2017, S. 724). Ermöglichen die Vorgaben den Mitgliedstaaten bei der Umsetzung oder Auslegung von Unionsrecht jedoch Spielräume, kann eine verfassungsrechtliche Prüfung nationaler Ausgestaltungen auch anhand des Grundgesetzes erfolgen (Hoidn, in: Roßnagel 2018, S. 61; BVerfG 2010; Starke 2017, S. 725). Im Ergebnis sind somit zwei verschiedene Normkomplexe zum Grundrechtsschutz sowie nationales Recht und Unionsrecht bei der Gestaltung eines EMS zu berücksichtigen. 


\subsubsection{Verfassungsrechtliche Vorgaben und Anforderungen}

Mittels der Chancen und Risiken eines EMS können die dabei relevanten Grundrechte anhand des Einflusses auf ihre Verwirklichungsbedingungen bestimmt werden. Aus ihren Rechtszielen können soziale Anforderungen abgeleitet und auf die Systemgestaltung übertragen werden, sodass für den Einsatz und die Gestaltung des Systems rechtliche Anforderungen hergeleitet werden können.

\subsubsection{Geförderte Grundrechte}

Der Einsatz eines EMS fördert eine autonome und flexible Arbeitszeitgestaltung, eine einfachere Trennung zwischen Arbeits- und Freizeit und eine Verbesserung der WorkLife-Balance, eine bessere Kontrolle über die eigene Erreichbarkeit, das Durchsetzen von Unternehmensregelungen und kann in der Konsequenz die Beschäftigtenzufriedenheit erhöhen sowie das Arbeitsklima und die Produktivität optimieren. Diese Chancen wirken sich positiv auf die allgemeine Handlungsfreiheit aus Art. 2 Abs. 1 i. V. m. Art. 1 Abs. 1 GG, die Berufsfreiheit und wirtschaftliche Betätigungsfreiheit gemäß Art. 12 Abs. 1 Satz 1 GG und die unionsrechtlichen Entsprechungen in Art. 15 Abs. 1 und Art. 16 GRCh, das allgemeine Persönlichkeitsrecht gemäß Art. 2 Abs. 1 i. V. m. Art. 1 Abs. 1 GG, das Recht auf Achtung des Privatlebens i. S. v. Art. 7 GRCh sowie das Recht auf körperliche Unversehrtheit gemäß Art. 2 Abs. 2 Satz 1 GG sowie das Recht auf körperliche und geistige Unversehrtheit gemäß Art. 3 Abs. 1 GRCh aus.

Die allgemeine Handlungsfreiheit schützt jedes menschliche Verhalten im Sinne des aktiven Elements der Entfaltung der Persönlichkeit (BVerfG 1980). Hierzu zählt jedes Verhalten, das für den Handelnden eine gesteigerte Relevanz für seine Persönlichkeitsentfaltung und Selbstverwirklichung hat (Murswiek, in: Sachs 2011: Art. 2 Rn. 49). Geschützt ist somit die freie Entscheidung über Tun und Unterlassen sowie das darauf beruhende Verhalten (Kube, in: Isensee und Kirchhof 2009: § 148 Rn. 49). Daneben gewährleistet die Berufsfreiheit das Recht zur allgemeinen, selbstbestimmten Betätigungsfreiheit und ermöglicht es, jede erlaubte Tätigkeit zu ergreifen und auszuüben (Panzer-Heemeier, in: Grobys und Panzer-Heemeier 2017: Persönlichkeitsrecht, Rn. 10). Davon umfasst sind Fragen der Arbeitsverteilung, -planung und allgemeinen Arbeitsorganisation sowie Handlungs- und Entscheidungsrechte im unternehmerischen Bereich (Ebd.; Schwarze, in: Schwarze et al. 2012: Art. 16 GRC, Rn. 3). Für den Arbeitgeber äußert sich das Grundrecht ferner in der Organisationsfreiheit. Es liegt daher in seiner Entscheidungsbefugnis die betriebliche Organisation eigenverantwortlich zu gestalten (Breuer, in: Isensee und Kirchhof 2010: $§ 170$ Rn. 88) und in seinem Unternehmen ein EMS einzuführen. Dadurch kommt er seiner Verpflichtung nach, den Gesundheitsschutz bei der Festlegung von betrieblichen Regelungen zu beachten und seine Mitarbeiter vor Überbeanspruchungen jeglicher Art zu schützen (vgl. BAG 1999: 2206 f.). Das Recht auf körperliche Unversehrtheit umfasst die menschliche Gesundheit im biologisch-physiologischen Sinn sowie das psychische Wohlbefinden, sofern es sich dabei um im körperlichen Sinn vergleichbare Schmerzen handelt (Murswiek, in: Sachs 2011: Art. 2 Rn. 149). Das Unionsgrundrecht 
erstreckt sich daneben explizit auf die geistige Unversehrtheit. Geschützt ist demnach auch die geistige Gesundheit im Sinne der Gesamtheit kognitiver, emotionaler und kommunikativer Fähigkeiten eines Menschen (Borowsky, in: Meyer 2014: Art. 3 Rn. 1, 36). Dies ist vor allem vor dem Hintergrund von psychischen Erkrankungen in Zusammenhang mit beruflichem Stress - z. B. Burn-Out - von besonderer Bedeutung.

Das allgemeine Persönlichkeitsrecht schützt den Einzelnen vor einer möglichen Beeinträchtigung seiner immateriellen Integrität und Selbstbestimmung (Murswiek, in: Sachs 2011: Art. 2 Rn. 59). Es kennzeichnet ein weiter Schutzauftrag, sodass es vor allem geeignet ist den modernen Bedrohungen technischer Entwicklungen entgegenzuwirken. Vom Schutzbereich umfasst ist der autonome Bereich privater Lebensgestaltung, in dem der Mensch seine Individualität entwickeln und wahren kann (BVerfG 1973). In diesem Sinne adressiert auch das Unionsgrundrecht auf Achtung des Privatlebens einen schützenswerten Bereich, in dem jede Person für die Entfaltung der Persönlichkeit und ihrer Selbstbestimmung den nötige Freiraum haben soll (Wolff, in: Schantz und Wolff 2017: Rn. 31). Daher ist es wichtig, dass ein EMS den Systemnutzern mehr Handlungsoptionen und -freiräume eröffnet und es dadurch nicht nur die Entfaltungsmöglichkeiten im beruflichen, sondern auch im privaten Kontext verbessern kann.

\subsubsection{Gefährdete Grundrechte}

Neben einer Verbesserung der Grundrechtsverwirklichung ist der Einsatz eines EMS auch mit Risiken für die Entfaltung von Grundrechten verbunden. Diese äußern sich vor allem in einer möglichen Profilbildung und Überwachung der Beschäftigten, dem Verlust der Datenkontrolle und -sicherheit, der Informationsgewinnung über die Kommunikationsinhalte sowie einer falschen Behandlung eingehender Kommunikationsversuche aufgrund technischer Fehlfunktionen. Diese Risiken wirken sich negativ auf das Fernmeldegeheimnis gemäß Art. 10 Abs. 1 GG, das Recht auf Achtung des Privatlebens, das Recht auf Schutz personenbezogener Daten gemäß Art. $8 \mathrm{GRCh}$, die informationelle Selbstbestimmung und das Recht auf Vertraulichkeit und Integrität informationstechnischer Systeme aus Art. 2 Abs. 1 i. V. m. Art. 1 Abs. 1 GG, das allgemeine Persönlichkeitsrecht, die Berufsfreiheit und wirtschaftliche Betätigungsfreiheit, die unternehmerische Freiheit sowie die allgemeine Handlungsfreiheit aus.

Das Fernmeldegeheimnis sowie das Recht auf Achtung des Privatlebens schützen die Vertraulichkeit individueller nicht öffentlicher Kommunikation, die aufgrund der räumlichen Distanz zwischen den Kommunizierenden auf die Übermittlung durch Dritte angewiesen ist, und dienen damit der freien Entfaltung der Persönlichkeit durch einen Kommunikationsaustausch mittels Fernmeldeverkehr (BVerfG 2002, S. 35 f., 1992; Jarass 2016: Art. 7 Rn. 6). Zudem stellt auch das Zurückhalten, Verhindern oder Verzögern einer Kommunikation einen Grundrechtseingriff dar (Jarass 2016: Art. 7 Rn. 31). Dazu zählt daher auch die Übermittlung von Kommunikationsinhalten und das Verzögern von Kommunikationsversuchen mittels eines EMS auf einem Smartphone. Geschützt ist neben dem verwendeten Kommunikationsmedium insbesondere der Kommunikationsinhalt, der sowohl privater als auch geschäftlicher Natur sein kann (BVerfG 2002, S. 36 f., 1992; Jarass 2016: 
Art. 7 Rn. 6 und 31). Im Sinne der informationellen Selbstbestimmung sowie dem Recht auf Schutz personenbezogener Daten hat der Einzelne zudem die Befugnis, grundsätzlich selbst Herr über seine persönlichen Daten zu sein und über die Preisgabe und Verwendung dieser zu bestimmen (BVerfG 1983; Kingreen, in: Calliess und Ruffert 2016: Art. 8 GRCh, Rn. 9). Als personenbezogene Daten gelten gemäß Art. 4 Nr. 1 der DatenschutzGrundverordnung (DSGVO) alle Informationen, die sich auf eine identifizierte oder identifizierbare natürliche Person beziehen. Identifizierbar ist eine Person, wenn sie direkt oder indirekt identifiziert werden kann. Davon umfasst sind zum einen solche Informationen, die der Privatsphäre oder der Intimsphäre der Person zuzuordnen sind, sowie zum anderen personenbezogene Daten, die beispielsweise den Beruf betreffen (Jarass 2016: Art. 8 Rn. 6; Bernsdorff, in: Meyer 2014: Art. 8 Rn. 13). Art. 8 GRCh normiert explizit das Recht auf Schutz personenbezogener Daten. Damit die freie Entfaltung der Persönlichkeit im Zuge der modernen Bedingungen elektronischer Datenverarbeitung gewährleistet werden kann, ist der Einzelne somit vor einer unbegrenzten Erhebung, Speicherung, Verwendung und Weitergabe seiner persönlichen Daten zu schützen (BVerfG 1983). Dies wirkt dem Gefühl der Systemnutzer, unter einer dauerhaften Beobachtung durch ihren Arbeitgeber zu stehen, entgegen. Nur dann sind die Nutzer in ihren Handlungen ungehemmt und können frei über diese entscheiden. Neben der Vertraulichkeit von Daten ist im Sinne des Rechts auf Vertraulichkeit und Integrität informationstechnischer Systeme schließlich auch die Integrität des Systems als solches zu gewährleisten.

\subsubsection{Vorgaben und Anforderungen des einfachen und europäischen Rechts}

Bei der Gestaltung eines EMS, das im Arbeitskontext zur Anwendung kommen soll, sind insbesondere die datenschutzrechtlichen Bestimmungen der DSGVO und des Bundesdatenschutzgesetzes (BDSG), des Arbeitszeitgesetzes (ArbZG) sowie des Betriebsverfassungsgesetzes (BetrVG) einzuhalten.

Gemäß Art. 5 Abs. 1 lit. a Var. 1 DSGVO müssen personenbezogene Daten auf rechtmäßige Weise verarbeitet werden. Daraus folgt für die Gestaltung eines EMS, dass dieses nur auf der Grundlage einer Einwilligung i. S. v. Art. 6 Abs. 1 Satz 1 lit. a DSGVO oder gesetzlichen Ermächtigung nach lit. b oder lit. c personenbezogene Daten verarbeiten darf. Daneben ergeben sich weitere Erlaubnistatbestände aus Art. 9 Abs. 2 und 88 DSGVO, dem Arbeitsvertrag oder bestehenden Betriebsvereinbarungen im Einsatzunternehmen. Ferner sind die Vorgaben des $\S 26$ Abs. 1 Satz 1 und Abs. 2 BDSG zu berücksichtigen.

Gemäß Art. 5 Abs. 1 lit. a Var. 3 DSGVO müssen personenbezogene Daten in einer für die betroffene Person nachvollziehbaren Weise verarbeitet werden. Eine intransparente Verarbeitung ist somit rechtswidrig. Demnach ist bei der Entwicklung eines EMS sicherzustellen, dass dieses den Grundsatz der Transparenz realisiert. Wichtig ist dabei, dass alle Informationen i. S. v. Erwägungsgrund (Eg.) 39 DSGVO leicht zugänglich, verständlich und in einfacher Sprache formuliert sind. Im Sinne seiner Entscheidungsfreiheit muss der 
Beschäftigte ferner über mögliche Folgen, die sich aus den Datenverarbeitungen ergeben, in Kenntnis gesetzt werden. Nur so kann er verstehen, welche Auswirkungen die Verarbeitung seiner personenbezogenen Daten durch das System haben wird und erleichtert ihm, eine Entscheidung bezüglich der Nutzung eines EMS zu treffen.

Gemäß Art. 25 Abs. 1 DSGVO muss der für die Datenverarbeitung Verantwortliche geeignete technische und organisatorische Maßnahmen (TOMs) treffen, um die Datenschutzgrundsätze aus Art. 5 Abs. 1 DSGVO wirksam umzusetzen. Dabei sind unter anderem die unterschiedlichen Eintrittswahrscheinlichkeiten und die Schwere der mit der Verarbeitung verbundenen Risiken für die Rechte und Freiheiten natürlicher Personen zu beachten. Zudem müssen die Maßnahmen gemäß Art. 5 Abs. 1 lit. f DSGVO eine angemessene Sicherheit für die verarbeiteten personenbezogenen Daten gewährleisten und sie vor unbefugter oder unrechtmäßiger Verarbeitung und vor unbeabsichtigtem Verlust, unbeabsichtigter Zerstörung oder Schädigung schützen. Je nach Einsatzunternehmen können sich demnach unterschiedlich notwendige Maßnahmen ergeben. Des Weiteren ist sicherzustellen, dass durch Voreinstellungen nur personenbezogene Daten, deren Verarbeitung für den jeweiligen bestimmten Verarbeitungszweck erforderlich ist, verarbeitet werden. Im Sinne des Zweckbindungsgrundsatzes aus Art. 5 Abs. 1 lit. b DSGVO müssen personenbezogene Daten für festgelegte, eindeutige und legitime Zwecke erhoben werden und dürfen nicht in einer mit diesen Zwecken nicht zu vereinbarenden Weise weiterverarbeitet werden. Die Zweckfestlegung und -bindung ist daher für die Datenverarbeitungstätigkeiten eines EMS ausschlaggebend. Jegliche Form der Datenverarbeitung für abstrakte und allgemeine Zwecke sowie die Verarbeitung von personenbezogenen Daten der Systemnutzer auf Vorrat für künftige, noch nicht absehbare Zwecke, ist rechtswidrig (Roßnagel, in: Simitis et al. 2019: Art. 5 DSGVO, Rn. 13). So können nur solche Informationen über den einzelnen Beschäftigten hierzu als geeignet angesehen werden, die einen Beitrag für die Zwecke der Erreichbarkeitssteuerung liefern oder diese fördern. Daran schließt der Grundsatz der Datenminimierung aus Art. 5 Abs. 1 lit. c DSGVO an, wonach personenbezogene Daten dem Zweck nach angemessen und erheblich sowie auf das für die Zwecke der Verarbeitung notwendige $\mathrm{Ma} \beta$ beschränkt sein müssen. Maßstab ist hierbei die Notwendigkeit und Unverzichtbarkeit der Daten, ohne die eingehende Kommunikationsversuche und somit die Erreichbarkeit der Systemverwender nicht gesteuert werden kann. Dahingehend muss der für die Verarbeitung Verantwortliche eine Speicherfrist festlegen, die auf das unbedingt erforderliche Mindestmaß beschränkt ist (vgl. Eg. 39 DSGVO). Zudem sind die personenbezogenen Daten i. S. d. Art. 5 Abs. 1 lit. e HS 1 DSGVO in einer Form zu speichern, die die Identifizierung der betroffenen Person nur so lange ermöglicht, wie dies für die Verarbeitungszwecke erforderlich ist.

Bei der Einführung eines EMS im Unternehmen sind schließlich auch arbeitsrechtliche Regelungen einzuhalten wie die Mitbestimmungsrechte des Betriebsrats gemäß $§ 87$ Abs. 1 Nr. 1 und 6 BetrVG. Ferner spielt in Zusammenhang mit der beruflichen Erreichbarkeit die werktägliche Arbeitszeit sowie die Ruhezeit eine große Rolle, da aus beruflichen Kommunikationsanfragen häufig die Erbringung von Arbeitsleistung resultiert (s. hierzu auch Kap. 2). Die Vorgaben zur Arbeitszeit können $§ 3$ ArbZG entnommen werden, 
wonach die werktägliche Arbeitszeit acht Stunden beträgt und unter bestimmten Bedingungen auf zehn Stunden verlängert werden darf. Zudem ist bei der Leistungserbringung gemäß $§ 5$ Abs. 1 ArbZG eine ununterbrochene Ruhezeit von mindestens elf Stunden nach Beendigung der täglichen Arbeitszeit einzuhalten, die gemäß Abs. 2 unter bestimmten Voraussetzungen und nur für bestimmte Berufsgruppen um eine Stunde verkürzt werden kann. Daneben können sich aus Tarif- oder Arbeitsverträgen, aber auch aus Betriebsvereinbarungen Abweichungen der täglichen Höchstarbeitszeit ergeben, wenn eine geringere werktägliche Arbeitszeit vereinbart wurde oder für bestimmte Berufsgruppen oder Personen in höheren beruflichen Positionen eine höhere Arbeitszeitgrenze zu berücksichtigen ist. Dies gilt insbesondere für leitende Angestellte, da für sie gemäß $\S 18$ Abs. 1 Nr. 1 ArbZG das Arbeitszeitgesetz keine Anwendung findet. Ferner kann unter bestimmten Voraussetzungen und für bestimmte Berufsgruppen die Ruhezeit um zwei Stunden verringert oder anderweitig angepasst werden. Um den gesetzlichen und betriebsbezogenen Anforderungen und dadurch vor allem dem Gesundheits- und Arbeitsschutz nachzukommen, sollte ein EMS die für die jeweilen Berufsgruppen bestehenden, aber auch die individuell festgelegten Vereinbarungen zur Arbeits- und Ruhezeit abbilden.

\subsection{Technische Anforderungen}

Die rechtlichen Vorgaben und Anforderungen können durch abstrakte technische Anforderungen präzisiert werden, indem die Architektur und die Grundfunktionen des Systems nach diesen Anforderungen ausgestaltet werden.

Die Chancen eines EMS können nur dann zum Tragen kommen, wenn das System die Erreichbarkeit seiner Nutzer effektiv steuern kann. Hierfür muss es korrekt und eigenständig eingehende Kommunikationsversuche behandeln und anschließend effektiv eine Entscheidung bezüglich ihrer Zustellung treffen. Ausschlaggebend ist somit die Zuverlässigkeit der spezifischen Zustellungs- und insbesondere der Entscheidungsfunktion des Systems. Verlässlich ist ein System, wenn es keine unzulässigen oder undefinierten Zustände annimmt und seinen vorgegebenen Anforderungen genügt (Bedner und Ackermann 2010, S. 327). Daher sollten technische Maßnahmen bei der Gestaltung des Systems ergriffen werden (z. B. redundante und entkoppelte Systemkomponenten), die Abweichungen bei den Funktionalitäten des Systems frühzeitig erkennen, um diese schnell beheben zu können und daraus resultierende mögliche Schäden zu vermeiden. Zudem ist anhand geeigneter TOMs die Zerstörung, Schädigung oder der Verlust der personenbezogenen Daten der Beschäftigten sowie ihre unrechtmäßige Kenntnisnahme durch Dritte auszuschließen. Anhaltspunkte für die Maßnahmenentwicklung bieten die Art. 25 und 32 DSGVO sowie der Stand der Technik. Dabei ist stets zu berücksichtigen, welches Risiko eines unbefugten oder unrechtmäßigen Zugriffs auf die Daten prognostiziert wird, welches Ausmaß ein solches Ereignis annehmen kann und welche Art von Daten durch das System verarbeitet werden (Reimer, in: Sydow 2018: Art. 5 Rn. 52). So fordert Art. 32 Abs. 1 HS 2 lit. a und lit. b DSGVO TOMs, die die Vertraulichkeit und Integrität im Zusammenhang 
mit der Verarbeitung personenbezogener Daten auf Dauer sicherstellen, sowie die Verschlüsselung personenbezogener Daten, um ein den Risiken angemessenes Schutzniveau zu ermöglichen. Dies kann beispielsweise durch das Implementieren von Zugriffsrechten und -berechtigungen, Authentifizierungsverfahren, Verschlüsselungssoftware, kryptografisch sicheren Hashfunktionen oder Protokollierung sichergestellt werden.

Damit das System den Erwartungen der Systemnutzer an die Vertraulichkeit der im System abgelegten Informationen gerecht werden und den Gefährdungen für deren Persönlichkeit entgegen wirken kann, sollte es so ausgestaltet werden, dass eine nicht zweckgemäße Verarbeitung der personenbezogenen Daten der Nutzer ausgeschlossen wird und nur die Daten erhoben werden, die zur Zweckerfüllung geeignet und erforderlich sind. Dies ist gemäß Art. 25 Abs. 2 Satz 1 DSGVO durch technische Voreinstellungen sicherzustellen. Alle darüberhinausgehenden Daten, die zwar dem Zweck dienen, der Zweck jedoch auch ohne sie erreicht werden kann, sind demnach zu löschen. Die entsprechend der Verarbeitungszwecke festgelegten Speicherfristen sind technisch zu implementieren, sodass die Daten nach der Zweckerreichung und nach Ablauf der gesetzten Fristen automatisch gelöscht werden oder die Identifizierung der betroffenen Person verhindert wird. Letzteres kann durch Pseudonymisierung oder Anonymisierung der Daten ermöglicht werden. So normieren Art. 25 Abs. 1 und Art. 32 Abs. 1 HS 2 lit. a DSGVO die Pseudonymisierung personenbezogener Daten als geeignete TOMs zum Schutz der Rechte und Freiheiten natürlicher Personen. Dadurch kann technisch sichergestellt werden, dass die zur Erreichbarkeitssteuerung und zur Bearbeitung der Kommunikationsversuche notwendigen personenbezogenen Daten der Beschäftigten diese nur solange identifizieren, wie dies zur Entscheidungsfindung des EMS erforderlich ist. Dabei sind nur solche Daten zur Erreichbarkeitssteuerung zu erheben und zu verwenden, die einen Beitrag zur technischen Eindämmung ständiger beruflicher Erreichbarkeit leisten. Dies ist schließlich auch auf die Speicherung dieser Daten zu übertragen, die entsprechend der Verarbeitungszwecke getrennt erfolgen sollte. Alle über den Zweck der Erreichbarkeitssteuerung hinausgehenden personenbezogenen Daten sind von der Verarbeitung auszuschließen, sodass eine lückenlose Überwachung des Nutzers ausgeschlossen ist.

Für den Beschäftigten wird durch den Gebrauch des EMS nicht ersichtlich sein, auf welcher detaillierten Grundlage dieses seine Entscheidungen trifft und an welcher Stelle die Verarbeitung seiner Daten erfolgt. Dieser Kontrollverlust wird verstärkt, wenn er keine Kenntnis darüber hat, welche Daten bei der Nutzung anfallen oder zu welchen Zwecken diese verwendet werden. Wichtig ist daher, dass er über jegliche mit der Systemnutzung verbundenen Funktionsweisen und Datenverarbeitungstätigkeiten informiert wird. Dies kann z. B. durch Hinweise innerhalb der App, durch Verweise auf Hyperlinks mit umfangreicheren Informationen, aber auch durch Hinweise auf unternehmensinternes Informationsmaterial erfolgen.

Schließlich ist technisch sicherzustellen, dass die Beschäftigten bei der Verwendung des EMS tatsächliche und nachvollziehbare Wahlmöglichkeiten erhalten, die ihnen ermöglichen, einfach und eigenständig Erreichbarkeitseinstellungen vorzunehmen. Auf diese Weise sind sie keinen technischen Zwängen ausgesetzt, die ihnen eine bestimmte 
Nutzungsweise auferlegen, sondern sie erhalten die Kontrolle über ihre Kommunikationssituation und sind frei in ihrer Entscheidung. Durch die verschiedenen Einstellungsoptionen des Systems, können sie zum Teil auch den Umfang der Datenverarbeitung steuern und erweitern, indem sie selbstbestimmt über die eigenen Kommunikationsumstände entscheiden. Die Anpassungsfähigkeit des Systems ist des Weiteren auch aufgrund der Vorgaben des Arbeits- und Betriebsverfassungsrechts sowie bestehender betrieblicher Regelungen oder Betriebsvereinbarungen des Einsatzunternehmens technisch zu gewährleisten. Bei der Entwicklung des Systems ist somit aufgrund unternehmensspezifischer Besonderheiten und möglicher Konfigurationswünsche des Betriebsrats ein hohes Maß an Anpassungsoptionen zu gewährleisten.

\subsection{Fazit}

Technische Systeme werden zu ganz bestimmten Zwecken entwickelt und dienen der Erfüllung bestimmter Ziele. Sie werden in unterschiedlichen gesellschaftlichen Bereichen eingesetzt und müssen innerhalb ihres Einsatzkontextes beurteilt werden. Die Sicherheit von Daten und der Schutz der von der Verarbeitung ihrer Daten betroffenen Personen ist dabei maßgeblich von der einzelnen Technologie abhängig. In der Regel ist zu beobachten, dass jedwede Probleme, die aus ihrem Gebrauch entstehen können, meist erst nach einem gewissen Nutzungszeitraum erkannt werden. Als Reaktion darauf soll das nachträgliche Einbauen von Schutzmechanismen in diese Technologien die Probleme beheben. Eine viel einfachere und praxistauglichere Lösung bietet die rechtliche Gestaltung eines technischen Systems, die begleitend zur Technikentwicklung erfolgen, aber auch bereits in einer konzeptionellen Phase der Entwicklung berücksichtigt werden kann. Diese Vorgehensweisen stellen sicher, dass der Einsatz der Technik nicht an rechtlichen Hürden scheitert oder Kosten im Nachgang, wenn ein System nicht rechtskonform gebaut wurde, vermieden werden. Zudem ist ein technischer Schutz von personenbezogenen Daten oder Unternehmensdaten meist effektiver als ein rechtlicher Schutz, denn wenn etwas technisch nicht möglich ist, ist es unmöglich ein darauf bezogenes rechtliches Verbot zu umgehen. Das Zusammenspiel zwischen Recht und Technik und somit die Zusammenarbeit zwischen den technischen Disziplinen und der Rechtswissenschaft ist maßgeblich, um der schnellen Entwicklung und zunehmende Verbreitung neuer technischer Innovationen zu begegnen, da sie zahlreiche Bereiche des gesellschaftlichen Lebens beeinflussen und verändern. 


\section{Literatur}

Arlinghaus, A., \& Nachreiner, F. (2013). When work calls - Associations between being contacted outside of regular working hours for work-related matters and health. Chronobiology International: The Journal of Biological \& Medical Rhythm Research, 30(9), 1197-1202.

Bundesarbeitsgericht (BAG). Urteil vom 19.01.1999. 1 AZR 499-98. Neue Juristische Wochenschrift, 1999(30): 2203-2207.

Bundesarbeitsgericht (BAG). Beschluss vom 26.08.2008. 1 ABR 16/07. Neue Zeitschrift für Arbeitsrecht, 2008(20): 1187-1194.

Bedner, M., \& Ackermann, T. (2010). Schutzziele der IT-Sicherheit. Datenschutz und Datensicherheit, 34(5), 323-328.

Bundesanstalt für Arbeitsschutz und Arbeitsmedizin (Hrsg.). (2017). Psychische Gesundheit in der Arbeitswelt. Wissenschaftliche Standortbestimmung. https://doi.org/10.21934/baua:bericht20170421.

Bundesverfassungsgericht (BVerfG). Urteil vom 05.06.1973. 1 BvR 536/72. BVerfGE 35, 202 (220).

Bundesverfassungsgericht (BVerfG). Beschluss vom 03.06.1980. 1 BvR 185/77. BVerfGE 54, 148 (153).

Bundesverfassungsgericht (BVerfG). Urteil vom 15.12.1983. 1 BvR 209, 269, 362, 420, 440, 484/83. BVerfGE 65, 1 (41ff.).

Bundesverfassungsgericht (BVerfG). Beschluss vom 25.03.1992. 1 BvR 1430/88. BVerfGE 85, 386 (396).

Bundesverfassungsgericht (BVerfG). Beschluss vom 09.10.2002. 1 BvR 1611/96, 1 BvR 805/98. BVerfGE 106, 28.

Bundesverfassungsgericht (BVerfG). Urteil vom 02.03.2010. 1 BvR 256, 263, 586/08. BVerfGE 125,260 (306).

Calliess, C., \& Ruffert, M. (Hrsg.). (2016). EUV/AEUV. München: C.H. Beck.

Daimler. (2014). Daimler Mitarbeiter können im Urlaub eingehende E-Mails löschen lassen. Pressemitteilung vom 13.08.2014. http://media.daimler.com/marsMediaSite/de/instance/ko/Daimler-Mitarbeiter-koennen-im-Urlaub-eingehende-E-Mails-loeschen-lassen.xhtml?oid=9919305. Zugegriffen am 13.09.2019.

Grobys, M., \& Panzer-Heemeier, A. (Hrsg.). (2017). Arbeitsrecht. Baden-Baden: Nomos.

Hammer, V., Pordesch, U., \& Roßnagel, A. (1993). Betriebliche Telefon- und ISDN-Anlagen rechtsgemäß gestaltet. Berlin/Heidelberg: Springer.

Hassler, M., Rau, R., Hupfeld, J., \& Paridon, H. (2016). iga.Report 23. Auswirkungen von ständiger Erreichbarkeit und Präventionsmöglichkeiten. Teil 2. https://www.iga-info.de/fileadmin/redakteur/Veroeffentlichungen/iga_Reporte/Dokumente/iga-Report_23_Teil2_Auswirkungen_staendiger_Erreichbarkeit.pdf. Zugegriffen am 16.09.2019.

Huber, M. O. (2016). Flexibel arbeiten in Zeit und Raum. In Bundesministerium für Arbeit und Soziales (Hrsg.), Werkheft 02. Wie wir arbeiten (wollen) (S. 88-93). https://www.bmas.de/SharedDocs/Downloads/DE/PDF-Publikationen/werkheft-02.pdf;jsessionid=EDE955817A0A2DC06FF0388A23380355?_blob=publicationFile\&v=2. Zugegriffen am 16.09.2019.

IG Metall. (2017). Die Befragung 2017. Arbeitszeit - sicher, gerecht und selbstbestimmt. https:// www.igmetall.de/download/docs_20170529_2017_05_29_befragung_ansicht_ komp_489719b89f16daca573614475c6ecfb706a78c9f.pdf. Zugegriffen am 16.09.2019.

Isensee, J., \& Kirchhof, P. (Hrsg.). (2009). Handbuch des Staats Rechts. Band VII Freiheitsrechte. Heidelberg: C.F. Müller.

Isensee, J., \& Kirchhof, P. (Hrsg.). (2010). Handbuch des Staats Rechts. Band VIII Grundrechte: Wirtschaft, Verfahren, Gleichheit. Heidelberg: C.F. Müller.

Jarass, H. D. (2016). Charta der Grundrechte der Europäischen Union. München: C.H. Beck. 
Laufs, U., Maucher, J., Miedzianowski, N., Rost, K., \& Saternus, Z. (2018). Erste Ergebnisse des Forschungsprojekts ,SANDRA“. Zeitschrift für Datenschutz-Aktuell, 06151.

Lindecke, C. (2015). Wem gehört die Zeit? Flexible Arbeitszeiten: Grenzenlose Freiheit oder grenzenlose Erreichbarkeit? Zeitschrift für Arbeitswissenschaft, 69(1), 31-38.

Maunz, T., \& Dürig, G. (Hrsg.). (2019). Grundgesetz-Kommentar. München: C.H. Beck.

Merten D. \& Papier H. J. (Hrsg) (2004). Handbuch der Grundrechte in Deutschland und Europa (Bd. I). Heidelberg: C.F. Müller.

Meyer, J. (Hrsg.). (2014). Charta der Grundrechte der Europäischen Union. Baden-Baden: Nomos.

Miedzianowski, N. (2017). S@NDRA: Neues Forschungsprojekt zur ständigen Erreichbarkeit in der digitalisierten Arbeitswelt. Zeitschrift für Datenschutz-Aktuell, 05558.

Miedzianowski, N., Saternus, Z., \& Staab, K. (2019). Stakeholderbezogene und rechtliche Anforderungen an ein Erreichbarkeitsmanagement-System. Zeitschrift für Datenschutz-Aktuell, 04381 und Zeitschrift für Datenschutz, 2019(11): XV-XIX.

Roßnagel, A. (Hrsg.). (2018). Das neue Datenschutzrecht. Europäische Datenschutz-Grundverordnung und deutsche Datenschutzgesetze. Baden-Baden: Nomos.

Sachs, M. (Hrsg.). (2011). Grundgesetz Kommentar. München: C.H. Beck.

Schantz, P., \& Wolff, H. A. (2017). Das neue Datenschutzrecht. München: C.H. Beck.

Schwarze, J., Becker, U., Hatje, A., \& Schoo, J. (Hrsg.). (2012). EU-Kommentar. BadenBaden: Nomos.

Simitis, S., Hornung, G., \& Spiecker gen. Döhmann, I. (Hrsg.). (2019). Datenschutzrecht. BadenBaden: Nomos.

Spiegel Online. (2011). VW-Betriebsrat setzt E-Mail-Stopp nach Feierabend durch. 23.12.2011. http://www.spiegel.de/wirtschaft/service/blackberry-pause-vw-betriebsrat-setzt-e-mail-stoppnach-feierabend-durch-a-805524.html. Zugegriffen am 13.09.2019.

Starke, C. P. (2017). Die Anwendbarkeit der Europäischen Grundrechtecharta auf rein nationale Gesetzgebungsakte. Deutsches Verwaltungsblatt, 132(12), 721-730.

Strobel, H. (2013). iga.Report 23. Auswirkungen von ständiger Erreichbarkeit und Präventionsmöglichkeiten.Teil 1.https://www.iga-info.de/fileadmin/redakteur/Veroeffentlichungen/iga_Reporte/ Dokumente/iga-Report_23_Staendige_Erreichbarkeit_Teil1.pdf. Zugegriffen am 16.09.2019.

Sydow, G. (Hrsg.). (2018). Europäische Datenschutzgrundverordnung. Baden-Baden: Nomos.

Open Access Dieses Kapitel wird unter der Creative Commons Namensnennung 4.0 International Lizenz (http://creativecommons.org/licenses/by/4.0/deed.de) veröffentlicht, welche die Nutzung, Vervielfältigung, Bearbeitung, Verbreitung und Wiedergabe in jeglichem Medium und Format erlaubt, sofern Sie den/die ursprünglichen Autor(en) und die Quelle ordnungsgemäß nennen, einen Link zur Creative Commons Lizenz beifügen und angeben, ob Änderungen vorgenommen wurden.

Die in diesem Kapitel enthaltenen Bilder und sonstiges Drittmaterial unterliegen ebenfalls der genannten Creative Commons Lizenz, sofern sich aus der Abbildungslegende nichts anderes ergibt. Sofern das betreffende Material nicht unter der genannten Creative Commons Lizenz steht und die betreffende Handlung nicht nach gesetzlichen Vorschriften erlaubt ist, ist für die oben aufgeführten Weiterverwendungen des Materials die Einwilligung des jeweiligen Rechteinhabers einzuholen.

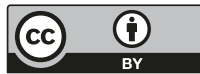

